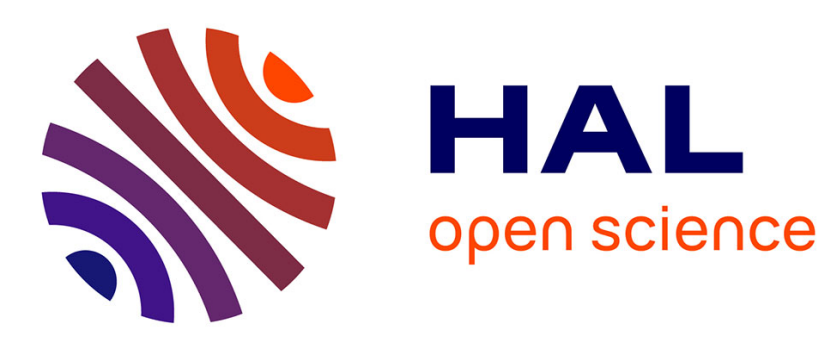

\title{
BERGE-VAISMAN AND NASH EQUILIBRIA: TRANSFORMATION OF GAMES
}

\author{
Antonin Pottier, Rabia Nessah
}

\section{To cite this version:}

Antonin Pottier, Rabia Nessah. BERGE-VAISMAN AND NASH EQUILIBRIA: TRANSFORMATION OF GAMES. International Game Theory Review, 2014, 16 (4), pp.166-175. 10.1142/S0219198914500091 . hal-01083736

\section{HAL Id: hal-01083736 https://hal.science/hal-01083736}

Submitted on 15 Dec 2016

HAL is a multi-disciplinary open access archive for the deposit and dissemination of scientific research documents, whether they are published or not. The documents may come from teaching and research institutions in France or abroad, or from public or private research centers.
L'archive ouverte pluridisciplinaire HAL, est destinée au dépôt et à la diffusion de documents scientifiques de niveau recherche, publiés ou non, émanant des établissements d'enseignement et de recherche français ou étrangers, des laboratoires publics ou privés. 


\title{
BERGE-VAISMAN AND NASH EQUILIBRIA: TRANSFORMATION OF GAMES
}

\author{
Antonin PotTiER \\ CIRED \\ CIRED-CNRS (UMR 8568) \\ 45 bis, avenue de la Belle Gabrielle \\ 94736 Nogent-sur-Marne Cedex \\ pottier@centre-cired.fr
}

\author{
Rabia NESSAH \\ IESEG School of Management \\ CNRS-LEM (UMR 8179) \\ 3 rue de la Digue \\ 59000 Lille, France \\ r.nessah@ieseg.fr
}

International Game Theory Review

Vol. 16, No. 4 (2014) 1450009 (8 pages)

DOI: $10.1142 / \mathrm{S} 0219198914500091$

\begin{abstract}
In this paper we reconsider the concept of Berge equilibrium. In a recent work, [Colman, A. M., Körner, T., Musy, O. and Tazdaït, T. [2011] Mutual support in games: Some properties of Berge equilibria, Journal of Mathematical Psychology 55, 166-175]. proposed a correspondence for two-player games between Berge and Nash equilibria by permutation of the utility functions. We define here more general transformations of games that lead to a correspondence with Berge and Nash equilibria and characterize all such transformations.
\end{abstract}

JEL codes: C72

keywords: Berge equilibrium; Nash equilibrium. 


\title{
BERGE-VAISMAN AND NASH EQUILIBRIA: TRANSFORMATION OF GAMES
}

\author{
Antonin Pottier, Rabiah Nessah \\ december 2013
}

\section{Introduction}

A burgeoning literature has focused on the Berge-Vaisman equilibrium (Abalo and Kostreva (2004, 2005); Colman et al. (2011); Courtois et al. (2011); Larbani and Nessah (2008); Musy et al. (2012); Nessah et al. (2007)). This concept captures the mutual support behavior in the normal form games. Furthermore, it is not a refinement of the Nash equilibrium. It thus fills a gap in the non-cooperative game theory.

Among different results that have been highlighted, there is one that will particularly come under scrutiny in this paper. Colman et al. (2011) established a correspondence between Berge-Vaisman and Nash equilibria. These authors show that the set of BergeVaisman equilibria of a two-player game is the set of Nash equilibria obtained by permutation of the utility functions of both players. In general, such a transformation may be feasible for one game, but it is then specific of the game. The aim of this paper is to examine whether there exists a general transformation of Berge-Vaisman equilibrium in terms of Nash equilibrium that could be valid not for one specific game, but for all games. Accordingly, we give all the possible transformations for two-player games. We also show that for $n$-player games, with $n>2$, there is no transformation that links Berge-Vaisman and Nash equilibria.

That a transformation between Nash and Berge-Vaisman equilibria may exist is not a fantasy from the authors. The concept of Berge-Vaisman equilibrium is sometimes dismissed, on the basis that it is simply Nash equilibrium "up to a transformation". The confusion may have arisen because in the most studied two-player case such a transformation exists. We clear away this confusion. When one is acquainted with Berge-Vaisman equilibrium, it is not surprising that a "transformation" does not exist. Together with precise definitions, we provide here a comprehensive proof of this fact.

\section{Berge-Vaisman Equilibrium and General Transformation}

Consider the following non cooperative game in normal form:

$$
G=\left\langle I,\left(S_{i}\right)_{i \in I},\left(u_{i}\right)_{i \in I}\right\rangle,
$$

where $I=\{1, \ldots, n\}$ denotes the set of players (with $n \geq 2$ ), $S_{i}$ the non-empty strategy set of player $i$, and $u_{i}$ her utility function. This utility function $u_{i}: S \rightarrow \mathbb{R}$ is defined on $S=\prod_{i \in I} S_{i}$, where $S$ is the set of all strategy profiles and $s_{-i}$ is the strategy profile $\left(s_{1}, \ldots s_{i-1}, s_{i+1} \ldots s_{n}\right) \in S_{-i}=\prod_{j \neq i} S_{j}$. We start with the definition of Nash and BergeVaisman equilibria. 
Definition 1 (Nash (1950)) A feasible strategy profile $\bar{s} \in S$ is said to be a Nash equilibrium of the game $G$ if, for any player $i \in I$, and any $s_{i} \in S_{i}$, we have:

$$
u_{i}\left(s_{i}, \bar{s}_{-i}\right) \leq u_{i}(\bar{s}) .
$$

A Nash equilibrium is defined as a strategy profile in which no agent, taking the strategies of the other players as given, wishes to change her strategy choice.

Definition 2 (Zhukovskii (1985)) A feasible strategy profile $\bar{s} \in S$ is a Berge-Vaisman equilibrium of the game $G$ if, for any player $i \in I$, and any $s_{-i} \in S_{-i}$, we have:

$$
u_{i}\left(\bar{s}_{i}, s_{-i}\right) \leq u_{i}(\bar{s}) .
$$

This definition means that, when a player $i \in I$ plays her strategy $\bar{s}_{i}$ from the BergeVaisman equilibrium $\bar{s}$, she obtains her higher utility when the remaining players willingly play the strategy $\bar{s}_{-i}$ from the Berge-Vaisman equilibrium.

These two equilibria are very different from one another. Yet, Colman et al. (2011) found a correspondence between the two equilibrium concepts. By linking the game $G=$ $\left\langle\{1,2\},\left(S_{1}, S_{2}\right),\left(u_{1}, u_{2}\right)\right\rangle$ to the game $\tilde{G}=\left\langle\{1,2\},\left(S_{1}, S_{2}\right),\left(v_{1}, v_{2}\right)\right\rangle$ with $v_{1}=u_{2}$ and $v_{2}=$ $u_{1}$, these authors proved the following theorem ${ }^{1}$.

Theorem 1 (Colman et al. (2011)) A strategy profile $\bar{s} \in S$ is a Berge-Vaisman equilibrium of the game $G$ if and only if it is a Nash equilibrium of the game $\tilde{G}$.

We recall here the proof of this theorem for sake of self-consistency.

Proof. This is a simple play with the definition, noting that $S_{-1}=S_{2}$ and $S_{-2}=S_{1}$.

Suppose $s^{*}$ is a Berge equilibrium of the game $G$. Then, by definition,

$$
u_{i}\left(s_{i}^{*}, s_{-i}\right) \leq u_{i}\left(s^{*}\right), \forall i \in N, \forall s_{-i} \in S_{-i},
$$

That is to say $u_{1}\left(s_{1}^{*}, s_{2}\right) \leq u_{1}\left(s^{*}\right), \forall s_{2} \in S_{2}$ and $u_{2}\left(s_{1}, s_{2}^{*}\right) \leq u_{2}\left(s^{*}\right), \forall s_{1} \in S_{1}$.

Since $u_{1}=v_{2}$ and $u_{2}=v_{1}, v_{1}\left(s_{1}, s_{2}^{*}\right) \leq v_{1}\left(s^{*}\right), \forall s_{1} \in S_{1}$ and $v_{2}\left(s_{1}^{*}, s_{2}\right) \leq v_{2}\left(s^{*}\right), \forall s_{2} \in$ $S_{2}$. Therefore

$$
v_{i}\left(s_{i}, s_{-i}^{*}\right) \leq v_{i}\left(s^{*}\right), \forall i \in N, \forall s_{i} \in S_{i}
$$

It follows that $s^{*}$ is a Nash equilibrium of the game $\tilde{G}$. The converse that if $s^{*}$ is a Nash equilibrium in $\tilde{G}$, then it is a Berge equilibrium in $G$ is proved in the same way, and the required result follows.

To propose a more general result, we introduce the following definition.

Definition 3 Let $F: \mathbb{R}^{n} \rightarrow \mathbb{R}^{n}$ be a function, $F$ is a transformation of the Berge-Vaisman equilibrium concept to the Nash equilibrium concept, if for all $n$-player games, the set of Berge-Vaisman equilibrium of the game

$$
G=\left\langle I,\left(S_{i}\right)_{i \in I},\left(u_{i}\right)_{i \in I}\right\rangle,
$$

is the same as the set of Nash equilibrium in the transformed game

$$
F(G)=\left\langle I,\left(S_{i}\right)_{i \in I},\left(F(u)_{i}\right)_{i \in I}\right\rangle,
$$

where the utility of player $i$ is $F_{i}\left(u_{1}, \ldots, u_{n}\right)$.

\footnotetext{
${ }^{1}$ Note that Larbani and Nessah (2008) identified another type of link. In particular, they showed that a Berge-Vaisman equilibrium can also be a Nash equilibrium under certain conditions.
} 
We call such an $F$ simply an $n$-transformation. The game $F(G)$ is called the transformed game of $G$. We can formulate the theorem of Colman et al. (2011) with this definition.

Theorem 2 (Theorem 1 restated) The function $F(x, y)=(y, x)$ is a 2-transformation.

The paper now characterizes all the $n$-transformations. We begin with a general lemma.

Lemma 1 Suppose that $F$ is an $n$-transformation $(n \geq 2)$, then $F_{i}$ does not depend on $u_{i}$.

Proof. We want to prove that for all $u_{-i} \in \mathbb{R}^{n-1}, F_{i}$ is constant in $u_{i}$. By symmetry, we put $i=1$. Suppose our lemma is false, then there exist $u_{-1}$ and $u_{1}, u_{1}^{\prime} \in \mathbb{R}$, such that $F_{1}\left(u_{1}, u_{-1}\right) \neq F_{1}\left(u_{1}^{\prime}, u_{-1}\right)$. Then we define the $n$-player game by the strategy set $S_{1}=\left\{s_{1}, s_{1}^{\prime}\right\}$ and $S_{-1}=\left\{s_{-1}\right\}$, and the utilities $u_{1}\left(s_{1}, s_{-1}\right)=u_{1}, u_{1}\left(s_{1}^{\prime}, s_{-1}\right)=u_{1}^{\prime}$ and $u_{-1}\left(s_{1}, s_{-1}\right)=u_{-1}, u_{-1}\left(s_{1}^{\prime}, s_{-1}\right)=u_{-1}$. The two strategy profiles of this game are BergeVaisman equilibria, however the transformed game has just one Nash equilibrium (it is $\left(s_{1}, s_{-1}\right)$ if $F_{1}\left(u_{1}, u_{-1}\right)>F_{1}\left(u_{1}^{\prime}, u_{-1}\right),\left(s_{1}^{\prime}, s_{-1}\right)$ if $\left.F_{1}\left(u_{1}, u_{-1}\right)<F_{1}\left(u_{1}^{\prime}, u_{-1}\right)\right)$. This is a contradiction with the assumption that $F$ is a transformation.

\subsection{The case $n=2$}

Now we restrict ourselves to the case $n=2$. According to the lemma, the transformation $F$ takes the form $F\left(u_{1}, u_{2}\right)=\left(F_{1}\left(u_{2}\right), F_{2}\left(u_{1}\right)\right)$.

Lemma 2 Suppose that $F$ is a 2-transformation, then $F_{1}$ is a strictly increasing function of $u_{2}$.

Proof. Suppose it is not the case. Then, there exist $u_{2}, u_{2}^{\prime} \in \mathbb{R}$ such that $u_{2}<u_{2}^{\prime}$ but $F_{1}\left(u_{2}\right) \geq F_{1}\left(u_{2}^{\prime}\right)$. Consider the 2-player game defined by the strategy set $S_{1}=\left\{s_{1}, s_{1}^{\prime}\right\}$ and $S_{2}=\left\{s_{2}\right\}$, and the utility $u_{1}\left(s_{1}, s_{2}\right)=u_{1}, u_{1}\left(s_{1}^{\prime}, s_{2}\right)=u_{1}$ and $u_{2}\left(s_{1}, s_{2}\right)=u_{2}, u_{2}\left(s_{1}^{\prime}, s_{2}\right)=$ $u_{2}^{\prime}$, where $u_{1}$ is any real number. The strategy profile $\left(s_{1}^{\prime}, s_{2}\right)$ is the unique Berge-Vaisman equilibrium, whereas in the transformed game $\left(s_{1}, s_{2}\right)$ is a Nash equilibrium (it is unique if $F_{1}\left(u_{2}\right)>F_{1}\left(u_{2}^{\prime}\right)$, if $F_{1}\left(u_{2}\right)=F_{1}\left(u_{2}^{\prime}\right)$, then $\left(s_{1}^{\prime}, s_{2}\right)$ is also a Nash equilibrium). This contradicts the assumption that $F$ is a transformation, i.e. that Nash equilibria of the transformed game are exactly the Berge-Vaisman equilibria of the original game.

Theorem 3 All the 2-transformations have the form $F\left(u_{1}, u_{2}\right)=\left(F_{1}\left(u_{2}\right), F_{2}\left(u_{1}\right)\right)$, where $F_{1}$ and $F_{2}$ are strictly increasing functions.

Proof. From the lemma 2, by symmetry, we have also proven that $F_{2}$ is a strictly increasing function of $u_{1}$. So the conditions of the theorem are necessary for $F$ to be a transformation. To prove that they are also sufficient, we proceed in two steps. By theorem 1, permuting the utility function of the two players is a 2-transformation. Then changing the utility of each player by increasing functions does not change the set of equilibria. So the functions with the form specified in the theorem are indeed transformations.

For two-player games, the concept of Berge-Vaisman equilibrium is reducible to the concept of Nash equilibrium, given that the utilities of the players be redefined. The above theorem precisely specifies the set of transformations for two-player games. Let us now move to the case where the number of players is greater than 2 . 


\subsection{The case $n>2$}

Let $F$ be an $n$-transformation. We have already proven that $F_{i}$ does not depend on $u_{i}$. We proceed to the reductio ad absurdum process by proving the following lemma.

Lemma 3 Suppose that $F$ is an n-transformation. Then $F_{1}$ does not depend on $u_{2}$.

Proof. Assuming the lemma is not valid, there exists $u_{-2}, u_{2}, u_{2}^{\prime} \in \mathbb{R}$, such that $F_{1}\left(u_{2}, u_{-2}\right) \neq$ $F_{1}\left(u_{2}^{\prime}, u_{-2}\right)$. We split $u_{-2}$ in $\left(u_{1}, u_{-1,-2}\right)$ so that $u_{-1,-2} \in \mathbb{R}^{n-2}$ (if $n=3$, this is just $\left.u_{3}\right)$. Consider the $n$-player game defined by the strategy set $S_{1}=\left\{s_{1}\right\}, S_{2}=\left\{s_{2}\right\}$, and $S_{-1,-2}=\left\{s_{-1,-2}, s_{-1,-2}^{\prime}\right\}$. The utilities for players other than 2 are constant $u_{1}=$ $u_{1}, u_{-1,-2}=u_{-1,-2}$, but for player $2 u_{2}\left(s_{1}, s_{2}, s_{-1,-2}\right)=u_{2}, u_{2}\left(s_{1}, s_{2}, s_{-1,-2}^{\prime}\right)=u_{2}^{\prime}$. Note that the definition of $u_{2}$ is valid precisely because $n>2$. In the transformed game, the two strategy profiles $\left(s_{1}, s_{2}, s_{-1,-2}\right)$ and $\left(s_{1}, s_{2}, s_{-1,-2}^{\prime}\right)$ are Nash equilibria, whereas in the initial game, only one strategy profile is a Berge-Vaisman equilibrium (it is $\left(s_{1}, s_{2}, s_{-1,-2}\right.$ ) if $u_{2}>u_{2}^{\prime}$ ). This contradicts our general assumption that $F$ is a transformation and proves the lemma.

We are now ready to show that an $n$-transformation cannot exist if $n>2$.

Theorem 4 No n-transformation exists when $n>2$.

Proof. In the proof of the previous lemma, the place of 2 was irrelevant, the only important thing was that there exists at least a third player (distinct from 1 and 2 ). So actually $F_{1}$ does not depend on any $u_{i}$ for all $i$ (recall our previous lemma). But it is irrelevant whether it is 1 or another player (the proof is the same if one replaces 1 by $i$ and 2 by $j$ ). So we have the result that $F$ is constant. But a constant cannot be a transformation, because there are games where not all strategy profiles are Berge-Vaisman equilibria (e.g. the game constructed in the proof of lemma 3).

\section{Illustration Example}

A key ingredient of the proof is the requirement of the transformation to be universable. If this condition is relaxed, a transformation may exist, as the example will show.

Example 1 Consider the following $n$-players (assume that $n>2$ ) on the unit square $S_{i}=[0,1]$, for each $i \in I=\{1, \ldots, n\}$. For player $i \in I$ and $s=\left(s_{1}, \ldots, s_{n}\right) \in S=[0,1]^{n}$, the payoff functions are:

$$
u_{i}(s)=\sum_{j=1}^{n} a_{i, j} s_{j},
$$

where $a_{i, j}$ is constant in $\mathbb{R}$, for each $i, j \in I$. This game is bounded, compact, convex, continuous and quasiconcave.

For each player $i \in I$, denote by $I_{-j}$ all players rather than player $j\left(I_{-j}=\{1, \ldots, j-\right.$ $1, j+1, \ldots, n\})$. The following proposition characterizes the existence of Berge-Vaisman equilibrium.

Proposition 1 The considered game possesses a Berge-Vaisman equilibrium if and only if for each $j \in I, \operatorname{sign}\left(a_{i, j}\right)=\operatorname{sign}\left(a_{h, j}\right),{ }^{2}$ for each $i, h \in I_{-j}$.

$$
{ }^{2} \operatorname{sign}(x)=\left\{\begin{array}{l}
+ \text { if } x \geq 0 \\
- \text { if } x \leq 0
\end{array}\right.
$$


Proof. Let $\bar{s} \in S$ be a Berge-Vaisman equilibrium of the game. Fix any $j \in I$, and let $i \in I_{-j}$. Since $\bar{s}$ is a Berge-Vaisman equilibrium, then

$$
u_{i}\left(\bar{s}_{i}, s_{-i}\right) \leq u_{i}(\bar{s}), \text { for each } s_{-i} \in S_{-i}
$$

Let $s_{-i}=\left(s_{j}, \bar{s}_{-\{i, j\}}\right)$ for any $s_{j} \in S_{j}$. This is possible, since $j \neq i$. Thus,

$$
a_{i, j} s_{j} \leq a_{i, j} \bar{s}_{j} \text {, for each } s_{j} \in S_{j} \text { and } i \neq j
$$

If $a_{i, j}>0$, then necessarily $\bar{s}_{j}=1$. If $a_{i, j}<0$, then necessarily $\bar{s}_{j}=0$. If $a_{i, j}=0$, there is no condition on $\bar{s}_{j}$. Obviously, $\bar{s}_{j}$ does not depend on $i \in I_{-j}$, therefore $\operatorname{sign}\left(a_{i, j}\right)=$ $\operatorname{sign}\left(a_{h, j}\right)$, for each $i, h \in I_{-j}$.

Conversely, suppose the conditions holds, then for $j \in I$ define $\bar{s}_{j}$ to the (common) sign of the $a_{i, j}, i \in I_{-j}$ (i.e. 0 is at least one of the $a_{i, j}$ is negative, 1 if at least one is positive, any number in $S_{j}$ if all are null). Then the strategy profile $\bar{s}$ is a Berge-Vaisman equilibrium of the game.

This fully characterizes the Berge-Vaisman equilibria of the game with linear utilities. We now do the same for the Nash equilibria.

Proposition 2 1) The game $\left\langle I, S,\left(u_{i}\right)_{i \in I}\right\rangle$ possesses an unique Nash equilibrium if and only if for each $i \in I, a_{i, i} \neq 0$.

2) The game $\left\langle I, S,\left(u_{i}\right)_{i \in I}\right\rangle$ possesses an infinity number of Nash equilibria if and only if there exists $i_{0} \in I$, so that $a_{i_{0}, i_{0}}=0$.

Proof. Since the game $\left\langle I, S,\left(u_{i}\right)_{i \in I}\right\rangle$ is compact, convex, continuous and quasiconcave, then it has a Nash equilibrium. Let $\bar{s} \in S$ be a Nash equilibrium of the game. Then for each $i$, and for each $s_{i} \in S_{i}$, we have $u_{i}\left(s_{i}, \bar{s}_{-i}\right) \leq u_{i}(\bar{s})$. By linearity of $u_{i}$, we obtain then

$$
a_{i, i} s_{i} \leq a_{i, i} \bar{s}_{i} \text {, for each } i \in I .
$$

If $a_{i, i}>0$, this is possible only if $\bar{s}_{i}=1$; if $a_{i, i}<0$, this is possible only if $\bar{s}_{i}=0$. If $a_{i, i}=0$, there is no condition on $\bar{s}_{i}$.

Conversely, define $\bar{s}_{i}$ according to the sign of $a_{i, i}$ (i.e. $\bar{s}_{i}=1$ when $a_{i, i}>0 ; \bar{s}_{i}=0$ when $a_{i, i}<0$; any $s_{i} \in S_{i}$ when $a_{i, i}=0$ ). Then the strategy profile $\bar{s}$ is a Nash equilibrium.

We can now give two examples of a transformation between Berge-Vaisman and Nash equilibrium.

Proposition 3 Consider the class of all games with linear utilities, such that for each $j \in I$, for $i, h \in I_{-j} \operatorname{sign}\left(a_{i, j}\right)=\operatorname{sign}\left(a_{h, j}\right)$. Restricts further the class to the game such that $a_{i, i}$ has the same sign of $a_{h, i}, h \neq i$, in a strict sense, that is $a_{i, i}$ is positive (resp. negative) if at least one $a_{h, i}, h \neq i$ is positive (resp. negative), $a_{i, i}$ is null if all $a_{h, i}, h \neq i$ are null. The identity is a transformation on this class.

Proof. The first condition ensures by Proposition 1 that a Berge-Vaisman equilibrium exists. The second condition ensures that Nash and Berge-Vaisman equilibrium are the same by the characterization of equilibrium strategy profiles of Propositions 1-2. So the identity is a transformation of game.

Let call $F$ the function that maps a vector $x \in \mathbb{R}^{n}$ to the diagonal vector of the sum of its components. 
Proposition 4 Consider the class of all games with linear utilities, such that for each $j \in I$, for $i, h \in I_{-j} \operatorname{sign}\left(a_{i, j}\right)=\operatorname{sign}\left(a_{h, j}\right)$ and $\left(\sum_{i} a_{i, j}\right)\left(\sum_{i \neq j} a_{i, j}\right)>0$. Then $F$ is a transformation on this class of games.

Proof. Take a game $G$ in the class. By the conditions that all signs of coefficients (off the diagonal) of a column are the same, there is a Berge-Vaisman equilibrium. This equilibrium is unique for not all the coefficients are null by the extra-condition. By Proposition 1, the Berge-Vaisman strategy profile is uniquely determined by the sign of the columns. The transformed game $F(G)$ still has linear utilities, with coefficients $b_{j, j}=\sum_{i} a_{i, j}$. The extracondition $\left(\sum_{i} a_{i, j}\right)\left(\sum_{i \neq j} a_{i, j}\right)>0$ ensures that $b_{j, j}$ has the same sign of the column $j$ of the original game. So the Nash equilibrium of the transformed game is the same as the Berge-Vaisman equilibrium of the original game.

\section{Conclusion}

In this paper we have characterized all the transformation of games between Nash and Berge-Vaisman equilibria. For 2-player games, Berge-Vaisman equilibrium is linked to Nash equilibrium by a permutation of utilities pertaining to changing utilities by strictly increasing functions. For $n$-player games, with $n>2$, the concept of Berge-Vaisman equilibrium is not transformable to the concept of Nash equilibrium by redefining the utilities of the players. This result stimulates further research on Berge-Vaisman equilibrium when there are more than two players.

\section{Acknowledgments}

The authors would like to thank Tarik Tazdaït for his advice and support, Vladimir Mazalov, Vladislav Zhukovskii and Arkady Kryazhimskiy for their helpful comments and suggestions.

\section{References}

Abalo, K. and M. Kostreva (2004) — Some existence theorems of Nash and Berge equilibria, Applied mathematics letters 17(5), pp. 569-573.

Abalo, K. and M. Kostreva (2005) - Berge equilibrium: some recent results from fixed-point theorems, Applied mathematics and computation 169(1), pp. 624-638.

Colman, A.M., T.W. Körner, O. Musy, and T. TazdaÏT (2011) - Mutual support in games: Some properties of Berge equilibria, Journal of Mathematical Psychology 55(2), pp. $166-175$.

Courtois, P., R. Nessah, and T. Tazdaït (2011) - How to play the games? Nash versus Berge behavior rules, Working Papers, LAMETA, Universtiy of Montpellier.

Larbani, M. and R. Nessah (2008) - A note on the existence of Berge and Berge-Nash equilibria, Mathematical Social Sciences 55(2), pp. 258-271.

Musy, O., A. Pottier, and T. Tazdaït (2012) - A New Theorem to Find Berge Equilibria, International Game Theory Review 14, pp. 1250005. 
NAsh, J.F. (1950) - Equilibrium points in n-person games, Proceedings of the national academy of sciences $3 \boldsymbol{6}(1)$, pp. 48-49.

Nessah, R., M. Larbani, and T. Tazdaït (2007) - A note on Berge equilibrium, Applied mathematics letters $20(8)$, pp. 926-932.

Zhukovskil, V.I. (1985) - Some Problems of Non-Antagonistic Differential Games, in P. Kenderov (Ed.), Matematiceskie metody v issledovanii operacij [Mathematical Methods in Operations Research], pp. 103-195. Sofia: Bulgarian Academy of Sciences. 